DOI 10.35381/cm.v7i1.560

\title{
El rol de la enfermera en pacientes críticos
}

\section{The role of the nurse in critical patients}

\author{
Maria Yolanda Garcia-Cedeño \\ mgarcia1216@hotmail.com \\ Universidad Estatal del Sur de Manabí, Jipijapa \\ Ecuador \\ https://orcid.org/0000-0001-5759-8953 \\ Delia Narcisa Quinche-Resabala \\ deki0592@gmail.com \\ Universidad Estatal del Sur de Manabí, Jipijapa \\ Ecuador \\ https://orcid.org/0000-0002-8945-1873 \\ Leonela Elizabeth García-Triviño \\ garciatrivinol@gmail.com \\ Universidad Estatal del Sur de Manabí, Jipijapa \\ Ecuador \\ https://orcid.org/ 0000-0001-6669-2204 \\ Carla-Carolina Zambrano-Santana \\ carlacarolina-89@hotmail.com \\ Universidad Estatal del Sur de Manabí, Jipijapa \\ Ecuador \\ https://orcid.org/0000-0001-8642-7643
}

Recibido: 15 de agosto de 2021

Aprobado: 01 de noviembre de 2021 


\title{
RESUMEN
}

La investigación tiene por objetivo analizar el rol de la enfermera en pacientes críticos en un hospital público de la provincia de Manabí - Ecuador. Se fundamentó en una investigación de tipo descriptiva. Dado que la significancia es ,423 siendo menor que 0,05 $(0,000<0,05)$, se acepta la $\mathrm{H} 1$ o hipótesis afirmativa y se rechaza $\mathrm{H} 0$ o nula, lo que indica que la relación del rol de enfermería y pacientes críticos fue significativa. En el cuidado de pacientes críticos se debe tener en cuenta el error humano, como parte de la atención al paciente crítico, por cuanto Los errores pueden causar graves daños a los usuarios de la salud y afectar cualitativa y cuantitativamente a las instituciones. Para tal fin, es necesario promover un trabajo en equipo eficaz, así como promover la debida dotación y formación del personal en razón de estar en disposición diligente de atender al paciente.

Descriptores: Coste de la vida; política de la salud; economía de la salud. (Palabras tomadas del Tesauro UNESCO).

\begin{abstract}
The research aims to analyze the role of the nurse in critical patients in a public hospital in the province of Manabí - Ecuador. It was based on a descriptive research. Given that the significance is .423 being less than $0.05(0.000<0.05)$, the $\mathrm{H} 1$ or affirmative hypothesis is accepted and $\mathrm{HO}$ or null is rejected, which indicates that the relationship between the role of nursing and critical patients was significant . In critical patient care, human error must be taken into account, as part of critical patient care, since Errors can cause serious harm to healthcare users and qualitatively and quantitatively affect institutions. To this end, it is necessary to promote effective teamwork, as well as to promote the proper staffing and training of staff because of their diligent disposition to care for the patient.
\end{abstract}

Descriptors: Cost of living; health policy; health economics. (Words taken from the UNESCO Thesaurus). 


\section{CIENCIAMATRIA}

Revista Interdisciplinaria de Humanidades, Educación, Ciencia y Tecnología

Año VII. Vol. VII. N¹. Edición Especial. 2021

Hecho el depósito de ley: pp201602FA4721

ISSN-L: 2542-3029; ISSN: 2610-802X

Universidad Nacional Experimental Francisco de Miranda (UNEFM). Santa Ana de Coro. Venezuela

Maria Yolanda Garcia-Cedeño; Delia Narcisa Quinche-Resabala; Garcia Triviño Leonela-Elizabeth;

Zambrano Santana Carla-Carolina

\section{INTRODUCCIÓN}

El rol de la enfermera en pacientes críticos es de suma importancia para concebir cuidados paliativos con efectividad con la procura de mantener con vida a la persona en la mejor calidad. Por lo tanto, es atractivo sacar provecho de las enfermeras en práctica avanzada para aumentar el acceso de los pacientes a cuidados intensivos y de emergencia, por consiguiente, se sugiere que la implementación de roles de enfermería de práctica avanzada en los entornos de emergencia y cuidados críticos mejora los resultados de los pacientes. La transformación de la prestación de servicios de salud mediante la utilización eficaz de la fuerza laboral puede aliviar el aumento inminente de la demanda de servicios de salud. Sin embargo, es necesario preparar primero un contexto receptivo para lograr un cambio sostenible (Woo et al. 2017).

Por otro lado, surge la necesidad de contar con enfermeras de cuidados críticos altamente capacitadas y con conocimientos continúa evolucionando hoy y lo hará en el futuro. Las demandas en medicina de cuidados críticos para enfermeras con títulos avanzados (maestría en enfermería: enfermera clínica especialista; o doctorado en enfermería: doctorado en filosofía en enfermería o doctor en práctica de enfermería) que trabajan en unidades de cuidados intensivos o unidades de cuidados progresivos pueden ayudar a enfrentar estos desafíos (Conley, 2019).

Por consiguiente, la certificación de la especialidad de enfermería se asoció con las creencias psicosociales individuales de las enfermeras y sus percepciones de las prácticas basadas en la evidencia en la unidad de cuidados intensivos, mientras que el nivel educativo no lo fue. Apoyar a las enfermeras en la obtención de la certificación de especialidad podría ayudar con la adopción de prácticas basadas en la evidencia como un medio para mejorar la calidad de la atención en la unidad de cuidados intensivos (Gigli et al. 2020).

Por otro lado, las enfermeras pediátricas desempeñan un papel importante en todas las fases de la recuperación de una lesión cerebral traumática (LCT), particularmente durante 


\section{CIENCIAMATRIA}

Revista Interdisciplinaria de Humanidades, Educación, Ciencia y Tecnología

Año VII. Vol. VII. N¹. Edición Especial. 2021

Hecho el depósito de ley: pp201602FA4721

ISSN-L: 2542-3029; ISSN: 2610-802X

Universidad Nacional Experimental Francisco de Miranda (UNEFM). Santa Ana de Coro. Venezuela

Maria Yolanda Garcia-Cedeño; Delia Narcisa Quinche-Resabala; Garcia Triviño Leonela-Elizabeth;

Zambrano Santana Carla-Carolina

la estadía en el hospital. Aunque se sabe que la práctica de enfermería basada en la evidencia mejora los resultados de los pacientes, existe una investigación limitada sobre el conocimiento y las creencias percibidas basadas en la evidencia de las enfermeras específicas para la atención de la LCT. Como se sabe que el conocimiento y las creencias percibidas por las enfermeras guían sus conductas de práctica, esta evaluación es importante para los resultados generales de la LCT. El propósito de este estudio fue evaluar el conocimiento y las creencias percibidas, basadas en la evidencia, de las enfermeras pediátricas al brindar atención a niños y adolescentes con TCE de moderado a grave (Oyesanya \& Snedden, 2018).

En resumen, las enfermeras atienden a pacientes críticos en diversos espacios hospitalarios, por consiguiente, la investigación tiene por objetivo analizar el rol de la enfermera en pacientes críticos en un hospital público de la provincia de Manabí Ecuador.

\section{MÉTODO}

La metodología se fundamentó en una investigación de tipo descriptiva correlacional con diseño no experimental de campo, lo cual permitió verificar la relación del rol de la enfermería y su efectividad en el cuidado de pacientes críticos.

La población tomada para la investigación se basó en 14 enfermeras de un centro de salud pública, del cual se reserva el nombre por condiciones éticas, quienes dieron su impresión sobre la variable de estudio, por medio de la encuesta como técnica y un instrumento tipo cuestionario de 21 ítems de cinco alternativas de respuestas en escala Likert.

Para la validez el contenido del instrumento se contó con el juicio de tres expertos, mientras que, para medir la confiabilidad, se empleó el cálculo del coeficiente de Alfa de Cronbach, del cual se obtuvo un resultado de 0,86 siendo considerable confiable para su aplicación. Los datos obtenidos fueron procesados mediante correlación de Spearman 
con apoyo del programa estadístico SPSS V25.

\section{RESULTADOS}

Se presentan los resultados obtenidos de la aplicación de la encuesta:

\section{Cuadro 1.}

Correlación entre rol de enfermeras y pacientes críticos.

\begin{tabular}{lrr}
\hline & Rol de enfermería & \multicolumn{1}{c}{ Pacientes críticos } \\
\hline Correlación de Pearson & 1 &, $423^{* *}$ \\
Sig. (bilateral) & 21 & 0 \\
$\mathrm{~N}$ & & 21 \\
\hline${ }^{* *}$ La correlación es significativa en el nivel 0,01 (bilateral). & \\
\hline
\end{tabular}

Fuente: Encuesta.

Dado que la significancia es ,423 siendo menor que $0,05(0,000<0,05)$, se acepta la $\mathrm{H} 1$ o hipótesis afirmativa y se rechaza $\mathrm{HO}$ o nula, lo que indica que la relación del rol de enfermería y pacientes críticos fue significativa.

\section{Prueba de hipótesis}

H1: la relación del rol de enfermería y pacientes críticos fue significativa.

H0: la relación del rol de enfermería y pacientes críticos no fue significativa.

\section{DISCUSIÓN}

Los resultados concuerdan con la investigación de (Pérez et al. 2020), al indicar que las demandas de atención al paciente, el entorno de práctica profesional y la falta de recursos para las familias dificultaron la participación del cuidador familiar de enfermería. Una 


\section{CIENCIAMATRIA}

Revista Interdisciplinaria de Humanidades, Educación, Ciencia y Tecnología

Año VII. Vol. VII. N¹. Edición Especial. 2021

Hecho el depósito de ley: pp201602FA4721

ISSN-L: 2542-3029; ISSN: 2610-802X

Universidad Nacional Experimental Francisco de Miranda (UNEFM). Santa Ana de Coro. Venezuela

Maria Yolanda Garcia-Cedeño; Delia Narcisa Quinche-Resabala; Garcia Triviño Leonela-Elizabeth;

Zambrano Santana Carla-Carolina

mayor atención a estas barreras en lo que respecta a la participación del cuidador familiar y los resultados clínicos debería ser una prioridad en la investigación futura.

Por otro lado, (Adams et al. 2017), plantea que la mayoría de las enfermeras de $\mathrm{UCl}$ consideraron la comunicación con las familias como una parte vital de su función y describieron comportamientos de apoyo. Sin embargo, percibieron barreras importantes para una comunicación eficaz; algunos como resultado de decisiones activas de su parte, y otros más allá de su control. Estas barreras a menudo hicieron que las enfermeras creyeran que las familias recibieron información y apoyo deficientes. El apoyo entre pares y la capacitación formal se identificaron como estrategias clave para superar las deficiencias.

Así mismo, un factor a tener en cuenta para que las enfermeras presten un servicio eficiente a los pacientes críticos, es el relacionado a las conductas de los jefes de unidad de enfermería afectan claramente el bienestar de las enfermeras de la UCI. Sin embargo, el papel de apoyar a las enfermeras de la $\mathrm{UCl}$ es complejo y desafiante. Se necesita más investigación para investigar las necesidades de las enfermeras de la $\mathrm{UCl}$ y los facilitadores y barreras que enfrentan los gerentes de las unidades de enfermería cuando apoyan el bienestar de las enfermeras en su unidad (Adams et al. 2019).

En complemento, (King et al. 2015), advierte que la organización del trabajo de las enfermeras, según modelos de atención reconocidos, puede tener un impacto significativo en el bienestar y desempeño de las enfermeras y los equipos de enfermería. Esta revisión se centra en dos modelos de prestación de cuidados de enfermería, a saber, la atención en equipo y total del paciente, y su efecto sobre el bienestar de las enfermeras.

Siendo considerable tener en cuenta el bienestar de las enfermeras, sobre todo cuando durante la pandemia de COVID-19, brindar atención a pacientes críticamente enfermos ha sido un desafío debido al número limitado de enfermeras capacitadas, la rápida transmisión del virus y el aumento de la agudeza del paciente en relación con el 


\section{CIENCIAMATRIA}

Revista Interdisciplinaria de Humanidades, Educación, Ciencia y Tecnología

Año VII. Vol. VII. N¹. Edición Especial. 2021

Hecho el depósito de ley: pp201602FA4721

ISSN-L: 2542-3029; ISSN: 2610-802X

Universidad Nacional Experimental Francisco de Miranda (UNEFM). Santa Ana de Coro. Venezuela

Maria Yolanda Garcia-Cedeño; Delia Narcisa Quinche-Resabala; Garcia Triviño Leonela-Elizabeth;

Zambrano Santana Carla-Carolina

virus. Estos factores han llevado a la implementación de la enfermería en equipo como modelo de atención de enfermería por necesidad de asignación de recursos. Las enfermeras pueden utilizar evidencia previa para informar el modelo de atención de enfermería y reimaginar las responsabilidades de atención del paciente durante una crisis (Beckett et al. 2021).

Siendo considerable tener en cuenta que las tres cuartas partes de las muertes en los hospitales están relacionadas con fallas en el trabajo en equipo y la comunicación. Los sistemas de atención aguda utilizan equipos de enfermeras registradas y asistentes de enfermería para la prestación primaria de atención de enfermería. Se han realizado investigaciones para mejorar la asociación entre la díada. Se necesitan revisiones de la literatura para sintetizar la efectividad de las intervenciones de delegación y comunicación entre enfermeras registradas y asistentes de enfermería sobre los resultados de los pacientes (Campbell et al. 2020).

Siendo considerable tener en cuenta el error humano, como parte de la atención al paciente crítico, por cuanto Los errores pueden causar graves daños a los usuarios de la salud y afectar cualitativa y cuantitativamente a las instituciones. Para los profesionales, el error suele estar relacionado con sentimientos de vergüenza, culpa y miedo al castigo, dada la cultura punitiva existente que se dirige a las omisiones y pierde la oportunidad de comprender y gestionar adecuadamente el error; Desde una perspectiva gerencial, es importante entender que los errores ocurren principalmente por problemas en el sistema organizacional, y no solo porque los profesionales cometen errores (Duarte et al. 2018). Por lo tanto, la seguridad del paciente en la percepción de los profesionales reflejó la importancia de la atención segura y la identificación de factores de riesgo en las condiciones laborales, predisponentes a errores. La comunicación de situaciones de riesgo, el desarrollo de la cultura de seguridad y la calificación son de suma importancia (Tomazoni et al. 2017). 


\section{CIENCIAMATRIA}

Revista Interdisciplinaria de Humanidades, Educación, Ciencia y Tecnología

Año VII. Vol. VII. N¹. Edición Especial. 2021

Hecho el depósito de ley: pp201602FA4721

ISSN-L: 2542-3029; ISSN: 2610-802X

Universidad Nacional Experimental Francisco de Miranda (UNEFM). Santa Ana de Coro. Venezuela

Maria Yolanda Garcia-Cedeño; Delia Narcisa Quinche-Resabala; Garcia Triviño Leonela-Elizabeth;

Zambrano Santana Carla-Carolina

Siendo considerable tener en cuenta el proceso de medicación es una tarea compleja y pueden ocurrir fallas en todas las etapas, desde la prescripción hasta la administración del medicamento. La enfermería actúa en las etapas de preparación y administración de medicamentos y debe cumplir con los nueve derechos, que son: paciente correcto, medicamento correcto, dosis correcta, momento correcto, ruta correcta, razón correcta, documentación correcta, educación del paciente correcta y respuesta correcta. Además, se deben aclarar dudas e identificar medicamentos con nombre del paciente, cama, nombre del medicamento, vía de administración, goteo y tiempo de infusión (Jirapaet et al. 2006).

En cuanto a pacientes neonatos, las enfermeras informaron que se habían perdido una variedad de actividades de atención al paciente en su último turno trabajado. Las enfermeras se perdían con mayor frecuencia las rondas, el cuidado bucal de los bebés ventilados, la educación y la participación de los padres en el cuidado y la alimentación bucal. La higiene de manos, la seguridad y la evaluación física y la administración de medicamentos fueron las que menos se pasaron por alto. Las razones más comunes para la atención perdida incluyeron interrupciones frecuentes, situaciones de pacientes urgentes y un aumento inesperado en el volumen y / o agudeza del paciente en la unidad (Tubbs-Cooley et al. 2015).

\section{CONCLUSIÓN}

En el cuidado de pacientes críticos se debe tener en cuenta el error humano, como parte de la atención al paciente crítico, por cuanto Los errores pueden causar graves daños a los usuarios de la salud y afectar cualitativa y cuantitativamente a las instituciones. Para tal fin, es necesario promover un trabajo en equipo eficaz, así como promover la debida dotación y formación del personal en razón de estar en disposición diligente de atender al paciente. 


\section{CIENCIAMATRIA}

Revista Interdisciplinaria de Humanidades, Educación, Ciencia y Tecnología

Año VII. Vol. VII. N¹. Edición Especial. 2021

Hecho el depósito de ley: pp201602FA4721

ISSN-L: 2542-3029; ISSN: 2610-802X

Universidad Nacional Experimental Francisco de Miranda (UNEFM). Santa Ana de Coro. Venezuela

Maria Yolanda Garcia-Cedeño; Delia Narcisa Quinche-Resabala; Garcia Triviño Leonela-Elizabeth;

Zambrano Santana Carla-Carolina

\section{REFERENCIAS CONSULTADAS}

Adams, A., Chamberlain, D., \& Giles, T. M. (2019). The perceived and experienced role of the nurse unit manager in supporting the wellbeing of intensive care unit nurses: An integrative literature review. Australian critical care : official journal of the Confederation of Australian Critical Care Nurses, 32(4), 319-329. https://doi.org/10.1016/j.aucc.2018.06.003

Adams, A., Mannix, T., \& Harrington, A. (2017). Nurses' communication with families in the intensive care unit - a literature review. Nursing in critical care, 22(2), 70-80. https://doi.org/10.1111/nicc.12141

Beckett, C. D., Zadvinskis, I. M., Dean, J., Iseler, J., Powell, J. M., \& Buck-Maxwell, B. (2021). An Integrative Review of Team Nursing and Delegation: Implications for Nurse Staffing during COVID-19. Worldviews on evidence-based nursing, 18(4), 251-260. https://doi.org/10.1111/wvn.12523

Campbell, A. R., Layne, D., Scott, E., \& Wei, H. (2020). Interventions to promote teamwork, delegation and communication among registered nurses and nursing assistants: An integrative review. Journal of nursing management, 28(7), 14651472. https://doi.org/10.1111/jonm.13083

Conley P. (2019). Certified and Advanced Degree Critical Care Nurses Improve Patient Outcomes. Dimensions of critical care nursing: DCCN, 38(2), 108-112. https://doi.org/10.1097/DCC.0000000000000342

Duarte, S., Stipp, M., Cardoso, M., \& Büscher, A. (2018). Patient safety: understanding human error in intensive nursing care. Revista da Escola de Enfermagem da U S $P$, 52, e03406. https://doi.org/10.1590/S1980-220X2017042203406

Gigli, K. H., Davis, B. S., Ervin, J., \& Kahn, J. M. (2020). Factors Associated With Nurses' Knowledge of and Perceived Value in Evidence-Based Practices. American journal of critical care : an official publication, American Association of Critical-Care Nurses, 29(1), e1-e8. https://doi.org/10.4037/ajcc2020866

Jirapaet, V., Jirapaet, K., \& Sopajaree, C. (2006). The nurses' experience of barriers to safe practice in the neonatal intensive care unit in Thailand. Journal of obstetric, gynecologic, and neonatal nursing : JOGNN, 35(6), 746-754. https://doi.org/10.1111/j.1552-6909.2006.00100.x 


\section{CIENCIAMATRIA}

Revista Interdisciplinaria de Humanidades, Educación, Ciencia y Tecnología

Año VII. Vol. VII. N¹. Edición Especial. 2021

Hecho el depósito de ley: pp201602FA4721

ISSN-L: 2542-3029; ISSN: 2610-802X

Universidad Nacional Experimental Francisco de Miranda (UNEFM). Santa Ana de Coro. Venezuela

Maria Yolanda Garcia-Cedeño; Delia Narcisa Quinche-Resabala; Garcia Triviño Leonela-Elizabeth;

Zambrano Santana Carla-Carolina

King, A., Long, L., \& Lisy, K. (2015). Effectiveness of team nursing compared with total patient care on staff wellbeing when organizing nursing work in acute care wards: a systematic review. $J B I$ database of systematic reviews and implementation reports, 13(11), 128-168. https://doi.org/10.11124/jbisrir-2015-2428

Oyesanya, T. O., \& Snedden, T. R. (2018). Pediatric nurses' perceived knowledge and beliefs of evidence-based practice in the care of children and adolescents with moderate-to-severe traumatic brain injury. Journal for specialists in pediatric nursing : JSPN, 23(2), e12209. https://doi.org/10.1111/jspn.12209

Pérez, A., Ramos, A., \& Carreras, G. (2020). Insulin Therapy in Hospitalized Patients. American journal of therapeutics, 27(1), e71-e78. https://doi.org/10.1097/MJT.0000000000001078

Tomazoni, A., Rocha, P. K., Ribeiro, M. B., Serapião, L. S., Souza, S., \& Manzo, B. F. (2017). Perception of nursing and medical professionals on patient safety in neonatal intensive care units. Segurança do paciente na percepção da enfermagem e medicina em unidades de terapia intensiva neonatal. Revista gaucha de enfermagem, 38(1), e64996. https://doi.org/10.1590/1983$\underline{1447.2017 .01 .64996}$

Tubbs-Cooley, H. L., Pickler, R. H., Younger, J. B., \& Mark, B. A. (2015). A descriptive study of nurse-reported missed care in neonatal intensive care units. Journal of advanced nursing, 71(4), 813-824. https://doi.org/10.1111/jan.12578

Woo, B., Lee, J., \& Tam, W. (2017). The impact of the advanced practice nursing role on quality of care, clinical outcomes, patient satisfaction, and cost in the emergency and critical care settings: a systematic review. Human resources for health, 15(1), 63. https://doi.org/10.1186/s12960-017-0237-9

@2021 por los autores. Este artículo es de acceso abierto y distribuido según los términos y condiciones de la licencia Creative Commons Atribución-NoComercial-Compartirlgual 4.0 Internacional (CC BY-NC-SA 4.0)

(https://creativecommons.org/licenses/by-nc-sa/4.0/). 